\title{
Deubiquitinase USP9X promotes cell migration, invasion and inhibits apoptosis of human pancreatic cancer
}

\author{
LI LIU $^{1 *}$, DAN YAO ${ }^{2 *}$, PENGBO ZHANG ${ }^{1}$, WEICHAO DING ${ }^{1}$, XIUZHONG ZHANG $^{1}$, \\ CHONG ZHANG $^{1}$, SHUAI GONG ${ }^{1}$, YI ZHANG ${ }^{1}$, JIKE WANG ${ }^{1}$, TING SUN ${ }^{1}$ and ZEQIANG REN ${ }^{1}$ \\ ${ }^{1}$ Department of General Surgery, The Affiliated Hospital of Xuzhou Medical University, \\ Xuzhou, Jiangsu 221002; ${ }^{2}$ Department of Gastrointestinal Surgery, \\ Huai'an Second People's Hospital, Huaian, Jiangsu 223000, P.R. China
}

Received January 12, 2017; Accepted July 6, 2017

DOI: 10.3892/or.2017.6050

\begin{abstract}
Ubiquitin specific peptidase 9, X-linked (USP9X), a significant regulatory protease in protein ubiquitination, has been proven to act as a proto-oncogene in several types of cancers, such as cervix, colon, breast, brain and lung cancers. The prognosis of patients with pancreatic ductal adenocarcinoma (PDAC) is extremely poor due to its high invasive and metastatic abilities. Nevertheless, whether USP9X acts as a proto-oncogene or a tumor-suppressor gene in PDAC is controversial and the mechanism of metastasis remains unknown. The present study focused on the effect of USP9X on the migration, invasion and apoptosis of PADC cells. We analyzed the expression of USP9X in pancreatic cancer tissues of different pathologic grades by immunohistochemical analysis. USP9X expression in the pancreatic cancer tissues was markedly increased in contrast to that noted in the adjacent non-tumor tissues. USP9X expression in PANC-1 cells was downregulated after transfection of shRNA-USP9X. Knockdown of endogenous USP9X expression evidently inhibited the migration and invasion of PANC-1 cells, and promoted cell apoptosis. Meanwhile, expression levels of Snail, Twist, $\mathrm{N}$-cadherin and vimentin were downregulated. E-cadherin expression was negatively correlated with USP9X expression and the expression of survivin was also downregulated in the PANC-1 cells. In brief, USP9X promoted the migration and invasion of PANC-1 cells probably by provoking epithelialmesenchymal transition, and also inhibited apoptosis. We believe that USP9X is a major oncogene that may play a significant role in the treatment and prognosis of PDAC.
\end{abstract}

Correspondence to: Professor Zeqiang Ren, Department of General Surgery, The Affiliated Hospital of Xuzhou Medical University, Xuzhou, Jiangsu 221002, P.R. China

E-mail: rzq0805@163.com

${ }^{*}$ Contributed equally

Key words: USP9X, pancreatic ductal adenocarcinoma, migration, invasion, apoptosis, epithelial-mesenchymal transition, survivin

\section{Introduction}

Pancreatic neoplasms are highly malignant tumors and a major cause of cancer-related mortality worldwide (1). The majority of pancreatic carcinomas are pancreatic ductal adenocarcinomas (PDACs). In recent years, the 5-year survival rate of PDAC patients has not significantly improved, and remains at $\sim 6 \%$ (2). The prognosis of PDAC is extremely poor, which is mostly ascribed to the fast growth, high migration, invasiveness and recurrence rates (3). To improve the efficiency of pancreatic cancer treatment, researchers must aim to understand the mechanisms underlying the migration, invasion and apoptosis of PDAC.

Ubiquitin specific peptidase 9, X-linked (USP9X), a deubiquitination enzyme, is a multifunctional posttranslational modifier that regulates many aspects of cell physiology $(4,5)$, such as DNA repair, and regulation of cell cycle and several signaling pathways. USP9X expression constantly changes in tumor progression $(6,7)$. Abnormal USP9X expression has been confirmed in several neoplasms, including lymphoma (8), colorectal cancer (9) and hepatocellular carcinoma (10). However, whether USP9X acts as a proto-oncogene or tumor-suppressor gene in pancreatic cancer cells is still controversial. Cox et al reported that USP9X possesses growth promotor functions in several established pancreatic cell lines (11), while Pérez-Mancera et al states that USP9X acts as a tumor-suppressor gene at the early stage of PDAC formation in mice (12). Thus, the concrete mechanism remains unclear as to how USP9X regulates PDAC development. In the present study, we discussed the effect of USP9X on epithelial-mesenchymal transition (EMT) in PANC-1 cells, aiming to understand its effect on cell migration and invasion. In terms of apoptosis, we observed the effect of USP9X downregulation on survivin expression.

EMT is a bio-process in which epithelial cells are transformed via specific procedures into a mesenchymal phenotype, and it is the molecular basis underlying the occurrence, invasion and metastasis of tumors (13). EMT plays an important role in the metastasis and invasion of neoplasms (14). It has been confirmed that EMT can be promoted by Snail and Twist genes, which induce the migration and invasion of tumor cells $(15,16)$. However, the 
specific connection between USP9X and EMT in PDAC and the effect of USP9X on PDAC remain poorly understood.

Survivin, which is highly inhibitory against cell apoptosis, was first identified at Altieri Laboratory of Yale University in 1997 by screening a human genomic library using effector cell protease receptor-1 (17). The bio-functions of survivin mainly involve the regulation of the cell cycle and stress response, mitosis promotion, cell and vascular proliferation, apoptosis inhibition and cancer cell autophagy regulation (18). However, high survivin expression has been detected in several tumors (19). Liu et al found that survivin is degraded by the ubiquitin proteasome pathway (20). Yet, the relationship between survivin and USP9X in pancreatic cancer is also unknown.

In the present study, we demonstrated that USP9X acts as a tumor metastasis supporter in PANC-1 cells, and downregulation of USP9X expression inhibited the migration and invasion of PANC-1 cells, while high expression of USP9X inhibited the apoptosis of PANC-1 cells. Therefore, USP9X functioned as an oncogene in PANC-1 cells and is closely related with the expression of Snail, Twist and survivin.

\section{Materials and methods}

Patients and samples. Tumor and paired adjacent nontumor tissues for immunohistochemical (IHC) analysis were obtained from 55 patients with malignant pancreatic tumors, who underwent surgical resection at The Affiliated Hospital of Xuzhou Medical University between 2009 and 2012 (Table I). None of the patients received chemotherapy or radiotherapy before surgery. Written informed consent was received from all patients or relevant family members. The present study was approved by the Ethics Committee of The Affiliated Hospital of Xuzhou Medical University.

Cell culture and treatment. PANC-1, a human pancreatic cancer cell line, was provided by Professor Changqing $\mathrm{Su}$ (Shanghai Oriental Hepatic Hospital of China). The cells were first cultured in Dulbecco's modified Eagle's medium (DMEM) containing 10\% fetal bovine serum (FBS) (both from HyClone, Shanghai, China), $100 \mu \mathrm{g} / \mathrm{ml}$ streptomycin and $100 \mathrm{U} / \mathrm{ml}$ penicillin. Then, the cells were routinely incubated in a humidified atmosphere at $37^{\circ} \mathrm{C}$ in $5 \% \mathrm{CO}_{2}$. The medium was refreshed every two days. Cell digestion and passage were conducted using $0.25 \%$ trypsin.

IHC analysis. Paraffin-embedded specimens were stained according to the kit manual. The primary antibody was anti-USP9X [1:1,000; Cell Signaling Technology (CST), Beverly, MA, USA]. In the IHC analysis, the staining intensity was rated as follows: 0, 1, 2 and 3 points: negative, weak, moderate and strong intensity, respectively (21). The percentage of positively-stained cells was rated as follows: $0,1,2,3$ and 4 points: $0,1-10,11-50,51-80$ and $>80 \%$, respectively. Then, the total score of immunoreactivity for each case was determined by multiplying the two sub-scores above. The average score from all five random fields at a magnification of x400 was used as the histological score. Tumors were categorized by the histological score into a negative group $(\leq 4)$ and a positive group (>4). The results were analyzed using Chi-square test.
shRNA transfection. USP9X-treated cells were plated into 6 -well plates $\left(3.0 \times 10^{5}\right.$ cells/well), for $24 \mathrm{~h}$ of adherence, and then transfected with shRNA (GenePharma, Shanghai, China; shRNA group) using SiLenFect (Bio-Rad Laboratories, Inc., Hercules, CA, USA). The sequence of shRNA was: GCTGCT AGGTTCCTCTTTCAAGAGAAGTAAAGAGGAACCTAG CAGCTT. In the vector group, the cells were transfected with NC-shRNA in the same way following the manufacturer's instructions. After $24 \mathrm{~h}$, the media were refreshed. After $48 \mathrm{~h}$, the cells were harvested for subsequent experiments.

Wound-healing assay. For the wound-healing assays, PANC-1 cells transfected according to the above-mentioned method were seeded in 6-well plates at a density of $2 \times 10^{6} /$ well. After $24 \mathrm{~h}$, the monolayers were scratched with a sterile pipette tip, followed by addition of serum-free medium. The sizes of the wounds were photographed at 0,12 , and $24 \mathrm{~h}$ separately. Each experiment was performed in triplicate.

Protein isolation and western blotting. The cultured PANC-1 cells were lysed by a modified radio-immunoprecipitation assay (RIPA) buffer (Shanghai, China) containing $0.5 \mathrm{M}$ pheylmethylsulfonyl fluoride (PMSF) and protease inhibitor cocktail (Complete Mini; Mannheim, Germany). Then, the homogenates were lysed on ice for $30 \mathrm{~min}$. The lysates were centrifuged at $4^{\circ} \mathrm{C}$ for $15 \mathrm{~min}$ and the supernatants were extracted. Total proteins were quantified with a bicinchoninic acid (BCA) protein assay kit (Pierce, Thermo Fisher Scientific, Inc., Waltham, MA, USA). For western blotting, protein samples $(50 \mu \mathrm{g})$ were loaded onto 8 or $12 \%$ sodium dodecyl sulfate-polyacrylamide gel electrophoresis (SDS-PAGE) and were then transferred onto polyvinylidene difluoride (PVDF) membranes (Millipore, Bedford, MA, USA). Then, the membranes were blocked with $5 \%$ non-fat milk at room temperature for $1 \mathrm{~h}$, and incubated with primary antibodies overnight at $4^{\circ} \mathrm{C}$, including anti-USP9X (1:1,000; CST), anti-survivin (1:200; Bioworld Technology, Nanjing, China), anti-Twist $(1: 1,000)$, anti-Snail $(1: 1,000)$, anti-N-cadherin $(1: 1,000)$, anti-vimentin $(1: 1,500)$, anti-E-cadherin $(1: 1,500)$ (all from ABclonal Inc., Cambridge, MA, USA) and anti- $\beta$-actin (1:1,000; Bioworld Technology). One day later, the membranes were washed with Tris-buffered saline and $0.05 \%$ Tween-20 (TBST), and then incubated with anti-rabbit or anti-mouse secondary antibody at room temperature for $1 \mathrm{~h}$. The intensities of the protein bands were assessed using ImageJ [National Institutes of Health (NIH), Bethesda, MD, USA].

Cell migration and invasion assays. The migration and invasion abilities of PANC-1 cells were assessed by cell migration and Matrigel invasion assays, respectively. After $24 \mathrm{~h}$ of transfection, the cells were suspended with serum-free medium and counted. In the assays, we furnished the invasion/migration chambers with 6.5-mm diameter tissue culture inserts and $8.0-\mu \mathrm{m}$ pore size polycarbonate membranes (Transwell; Corning, Corning, NY, USA USA). For invasion assays, Matrigel (1:6; BD Biosciences, Bedford, MA, USA) was blended with serum-free DMEM, and $50 \mu 1$ of the mixture was added into the chamber. The chambers were set into 24-well tissue culture plates, and then put in a sterile ultraclean bench overnight. On the next day, after the Matrigel 
was solidified, 5.0x10 4 PANC-1 cells were added to the upper chamber. The total volume was $200 \mu \mathrm{l}$, with adequate addition of the serum-free medium. For the migration assays, the operation method was the same as the invasion assays, except for the addition of Matrigel. In both trials, the lower chamber was filled with $600 \mu \mathrm{l}$ of $20 \%$ FBS. After $24 \mathrm{~h}$ of incubation at $37^{\circ} \mathrm{C}$ with $5 \% \mathrm{CO}_{2}, 90 \%$ paraformaldehyde was used to fix the cells invading the lower surfaces of the polycarbonate membranes. Then, the cells were stained with crystal violet and counted under a microscope. The cells were washed with phosphate-buffered saline (PBS) two times. Then, the water on the surfaces was gently wiped off and five views were taken for each insert.

Cell apoptosis assay. Cell apoptosis was detected by flow cytometry via a double staining method using an Annexin V-fluorescein isothiocyanate (FITC)-conjugated propidium iodide (PI) apoptosis kit (KeyGen Biotech. Co, Ltd., Nanjing, China). We performed the apoptosis assay after $48 \mathrm{~h}$ of transfection. PANC-1 cells were inoculated in 6-well plates at a density of $3 \times 10^{5}$ cells/ well. When the cells covered $\sim 50 \%$ of the 6 -well plates, the 6 -well plates were divided into a normal control (NC group), a negative control (vector group) and a shRNA-transfected group (shRNA group; transfected similarly as the above method). After $48 \mathrm{~h}$, the cells in the three groups were digested with $0.25 \%$ trypsin without ethylenediamine tetraacetic acid (EDTA; Invitrogen, Carlsbad, CA, USA). Then, the cells were washed with PBS for two times, (each $5 \mathrm{~min}$ ) and centrifuged at a speed of 5,000 rpm for $5 \mathrm{~min}$. Next, the cells were resuspended in $500 \mu \mathrm{l}$ of binding buffer and stained with $5 \mu \mathrm{l}$ Annexin V-FITC and $5 \mu \mathrm{l}$ of PI, for 15 to $20 \mathrm{~min}$ of reaction in a darkroom. Finally, the three groups of cells were stained, counted and analyzed using a flow cytometer (FACSCalibur; BD Biosciences).

CCK-8 assay for cell viability. Cells which were transfected for $48 \mathrm{~h}$ were plated in 96 -well plates with $5 \times 10^{3}$ cells/well. Six repetitive wells were prepared for each group. Control wells were created adding the same volume of culture medium. After $6,24,48,72$ and $96 \mathrm{~h}$ of incubation, the cells were then treated with $10 \mu \mathrm{l}$ Cell Counting Kit-8 (CCK-8) (Dojindo, Kumamoto, Japan) for another $2 \mathrm{~h}$ in an incubator at $37^{\circ} \mathrm{C}$. An automated microplate reader (Sunrise, Tecan, Switzerland) was applied to assess the OD values for each well at $450 \mathrm{~nm}$.

Statistical analysis. Data are expressed as mean \pm standard deviation (SD). Differences between groups were examined by Student's t-test or one-way analysis of variance (ANOVA). $\mathrm{P}<0.05$ and $\mathrm{P}<0.01$ were considered to indicate statistically significant results. All statistical analyses were performed using SPSS (version 13.0; SPSS, Inc., Chicago, IL, USA) and GraphPad Prism (version 5.0; GraphPad Software, Inc., La Jolla, CA, USA).

\section{Results}

USP9X is highly expressed in human pancreatic cancer tissues. IHC analysis revealed that USP9X expression was upregulated in 32 of 55 tumor tissues compared to the adjacent non-tumor tissues (Fig. 1A-C and Table I). Moreover, high expression of USP9X was markedly associated with stage, grade and lymph node metastasis (all $\mathrm{P}<0.05$ ) (Table I). The
Table I. Clinicopathological association of USP9X expression in pancreatic cancer tissues.

\begin{tabular}{|c|c|c|c|c|}
\hline \multirow[b]{2}{*}{ Variables } & \multirow[b]{2}{*}{$\begin{array}{l}\text { No. of } \\
\text { cases }\end{array}$} & \multicolumn{2}{|c|}{ USP9X expression } & \multirow[b]{2}{*}{ P-value } \\
\hline & & $\begin{array}{l}\text { High } \\
\mathrm{n}(\%)\end{array}$ & $\begin{array}{l}\text { Low } \\
\mathrm{n}(\%)\end{array}$ & \\
\hline Age (years) & & & & 0.803 \\
\hline$<60$ & 25 & $15(27.27)$ & $10(18.18)$ & \\
\hline$\geq 60$ & 30 & $17(30.09)$ & $13(23.63)$ & \\
\hline Sex & & & & 0.444 \\
\hline Male & 32 & $20(36.36)$ & $12(26.63)$ & \\
\hline Female & 23 & $12(26.63)$ & $11(20.00)$ & \\
\hline Tumor size $(\mathrm{cm})$ & & & & 0.262 \\
\hline$<4.0$ & 24 & $16(29.09)$ & $8(14.55)$ & \\
\hline$\geq 4.0$ & 31 & $16(29.09)$ & $15(27.27)$ & \\
\hline Grade & & & & 0.019 \\
\hline High & 7 & $2(3.64)$ & $5(9.09)$ & \\
\hline Middle & 34 & $18(32.73)$ & $16(29.09)$ & \\
\hline Low & 14 & $12(26.63)$ & $2(3.64)$ & \\
\hline $\begin{array}{l}\text { Lymph node status } \\
\text { (metastasis) }\end{array}$ & & & & 0.002 \\
\hline Yes & 20 & $17(30.90)$ & $3(5.45)$ & \\
\hline No & 35 & $15(27.27)$ & $20(36.36)$ & \\
\hline TNM stage ${ }^{a}$ & & & & 0.006 \\
\hline I & 25 & $9(16.36)$ & $16(29.09)$ & \\
\hline II & 28 & $22(40.00)$ & $6(10.91)$ & \\
\hline IV & 2 & $1(1.82)$ & $1(1.82)$ & \\
\hline
\end{tabular}

${ }^{a}$ Cancer staging was carried out according to the International Union against Cancer (UIAC) tumor-node-metastasis (TNM) system.

results imply that USP9X is positively correlated with the degree of tumor malignancy and may be closely related with pathophysiological progression of PDAC.

USP9X expression after transfection. First, we confirmed the alteration of the expression of USP9X. Thus, four shRNA sequences (purchased from GenePharma Co.) directed against USP9X were evaluated. As a control, an empty plasmid vector was transduced into PANC-1 cells. After $48 \mathrm{~h}$, the USP9X expression in PANC-1 cells was detected via western blotting. After filtration, we chose one group of plasmids with the best effect. The USP9X expression was significantly downregulated $(\mathrm{P}<0.01)$ (Fig. 2A and $\mathrm{B})$.

Depletion of USP9X inhibits cell migration and invasion. The functional effects of USP9X on pancreatic cancer cell migration and invasion abilities were examined via Transwell and wound healing assays. The suppression of USP9X expression significantly inhibited cell migration (Fig. 3A) and invasion ability (Fig. 3B). Next, we monitored the wound healing assays and similar results were found. The migration ability of the PANC-1 cells was significantly decreased when USP9X was suppressed (Fig. 3C). 


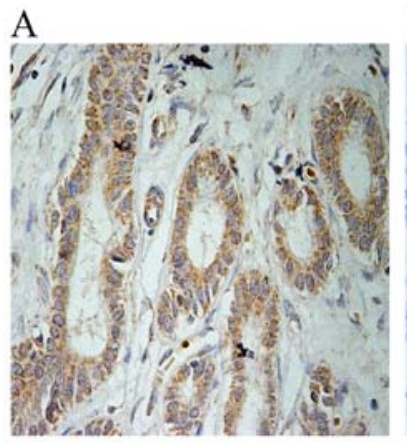

B

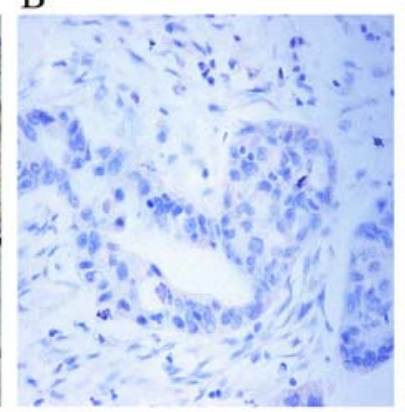

$\mathrm{C}$

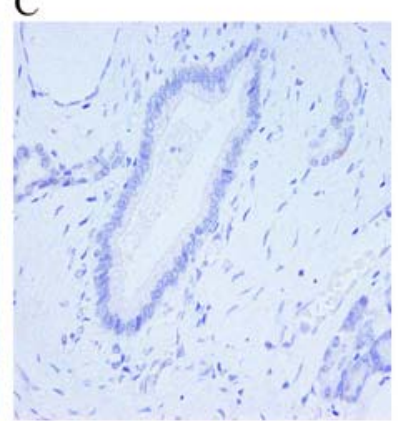

Figure 1. USP9X expression in tumor and adjacent non-tumor tissues (magnification, x400). (A) High expression of USP9X in pancreatic cancer tissues. (B) Low expression of USP9X in pancreatic cancer tissues. (C) Negative expression of USP9X in adjacent non-tumor tissues.

A

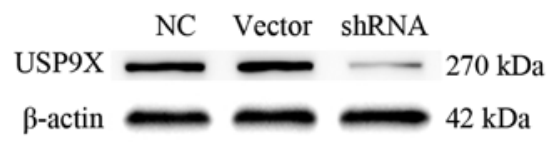

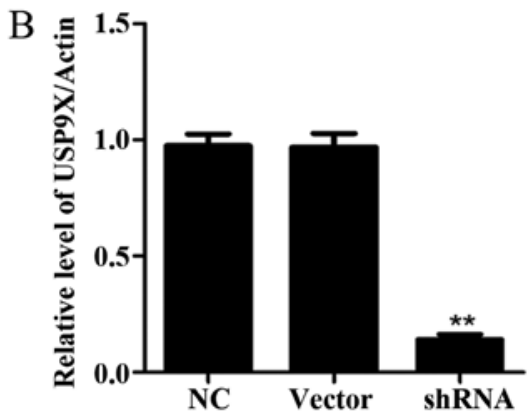

Figure 2. Decreased expression of USP9X after transfection of specific plasmids. Expression of protein levels of USP9X in PANC-1 cells after interference as detected by western blotting. (A) Western blotting. (B) Quantitative analysis of USP9X; ${ }^{* *} \mathrm{P}<0.01$.

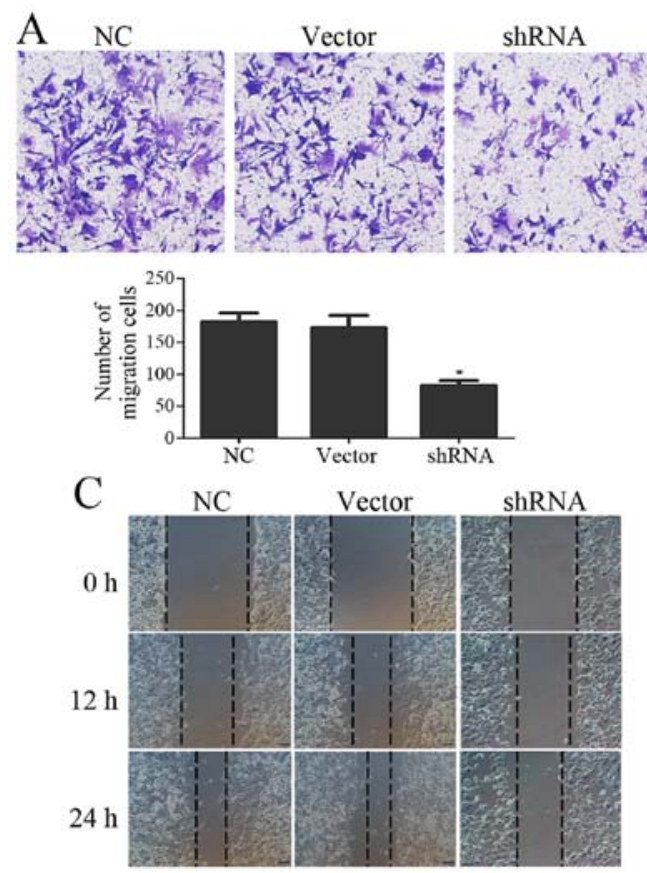

B
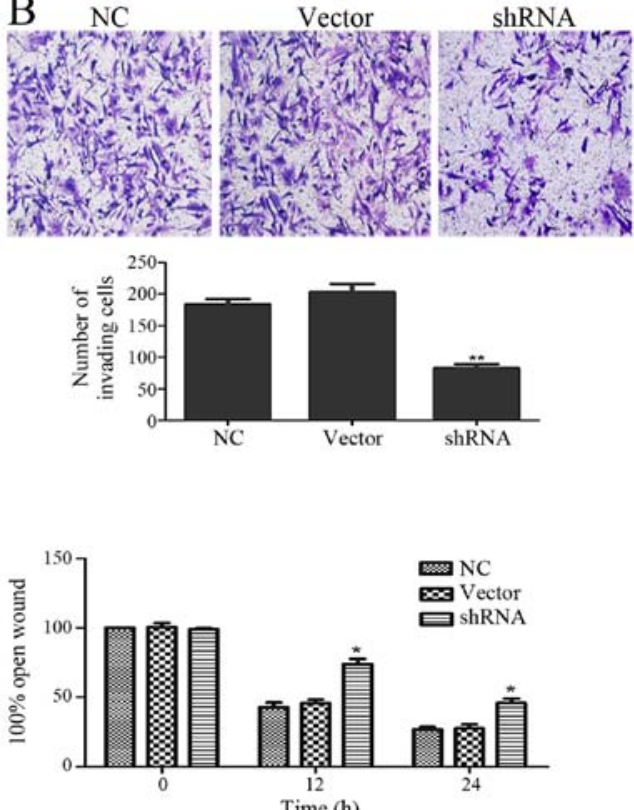

Figure 3. Downregulation of USP9X inhibits PANC-1 cell migration and invasion. (A) Cell migration assay was implemented after USP9X knockdown in the PANC-1 cells. The ability to migrate by Boyden chamber was significantly decreased compared with the vector and NC groups. The relative numbers of migrated cells of the three groups are shown below the images. (B) The PANC-1 cells transfected with USP9X-shRNA demonstrated significantly decreased cell invasion ability as observed from the cell invasion assay. The relative numbers of invasive cells of the three groups are shown below the images. (C) The PANC-1 cells transfected with USP9X-shRNA demonstrated significantly inhibited cell migration ability as determined by wound healing assay. The relative percentage of the open wound of the three groups is shown at the right. All experiments were carried out in triplicate. Data are shown as mean $\pm \mathrm{SD}$; ${ }^{*} \mathrm{P}<0.05{ }^{, * *} \mathrm{P}<0.01$.

Depletion of USP9X reverses EMT of PANC-1 cells. To explore the effects of USP9X on the EMT of PANC-1 cells, we examined the expression levels of mesenchymal cell markers ( $\mathrm{N}$-cadherin and vimentin) and epithelial cell marker 
A

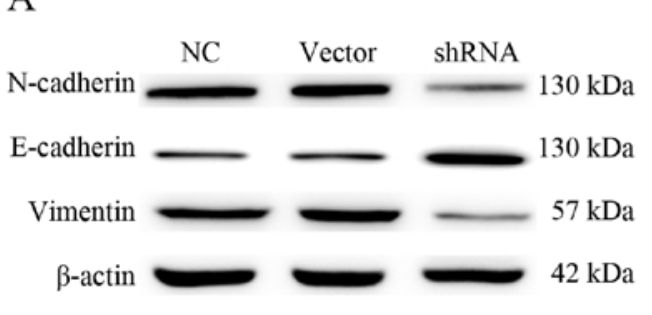

$\mathrm{C}$

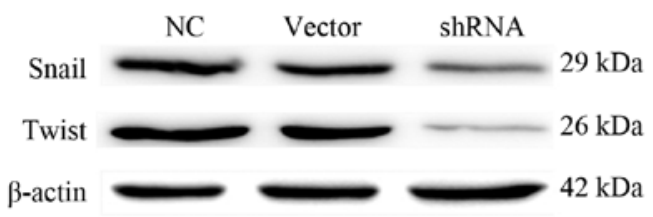

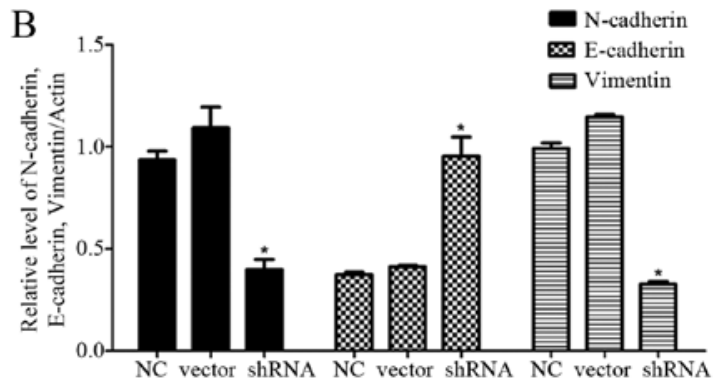

$\mathrm{D}$

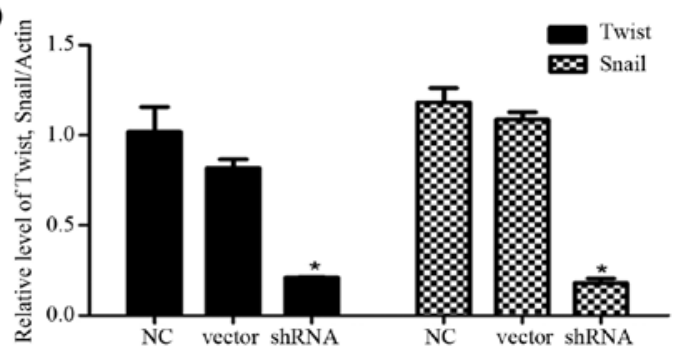

Figure 4. Depletion of USP9X reverses epithelial-mesenchymal transition of PANC-1 cells. (A) Western blot analysis of the relative protein levels of N-cadherin, vimentin and E-cadherin in USP9X-downregulated and control groups of PANC-1 cells. (B) Quantitative analysis of relative protein levels of N-cadherin, vimentin and E-cadherin in PANC-1 cells. (C) Western blot analysis of the relative protein levels of Snail and Twist in USP9X-downregulated and control groups of PANC-1 cells. (D) Quantitative analysis of relative protein levels of Snail and Twist in PANC-1 cells. Experiments were carried out in triplicate. Data are shown as mean $\pm \mathrm{SD} ;{ }^{*} \mathrm{P}<0.05$.
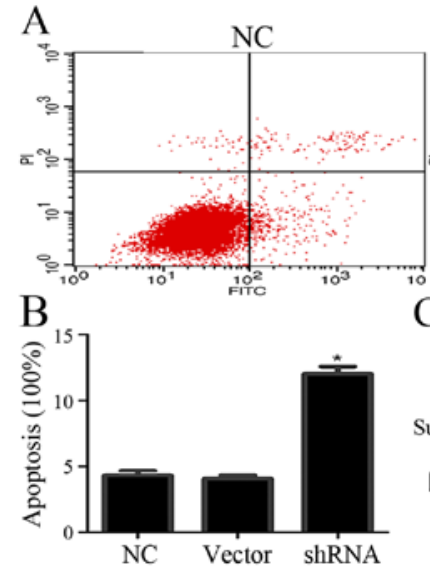

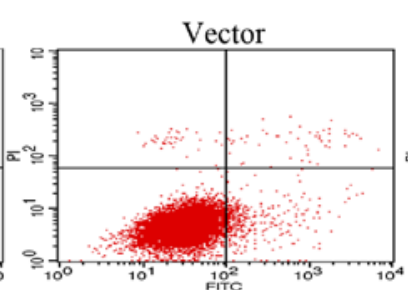

$\mathrm{C}$
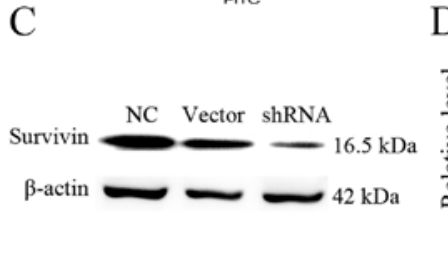

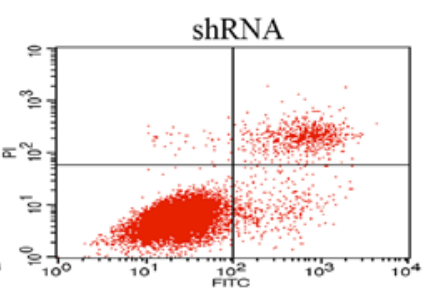

$\mathrm{D}$

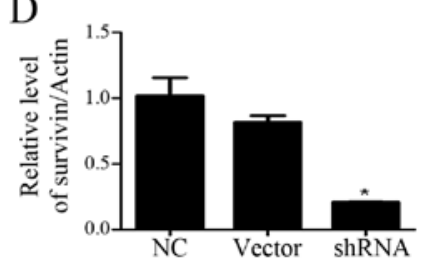

Figure 5. Inhibition of USP9X induces apoptosis. (A and B) After the PANC-1 cells were transfected with USP9X-shRNA and vector, cell apoptosis was detected using flow cytometry. The apoptotic rate was statistically significantly increased in the USP9X-silenced cells. (C) Western blot analysis of the relative protein level of survivin in the USP9X-silenced and control groups of PANC-1 cells. (D) Quantitative analysis of the relative protein level of survivin in PANC-1 cells. Experiments were carried out in triplicate. Data are shown as mean $\pm \mathrm{SD} ;{ }^{*} \mathrm{P}<0.05$.

(E-cadherin) by western blotting. The results showed that the downregulation of USP9X expression significantly reduced the expression of $\mathrm{N}$-cadherin and vimentin compared with these levels in the control groups $(\mathrm{P}<0.05$; Fig. $4 \mathrm{~A}$ and $\mathrm{B})$. In contrast, the E-cadherin expression was increased $(\mathrm{P}<0.05$; Fig. 4A and B).

To further confirm the effect of USP9X on the EMT of PANC-1 cells, we determined the expression levels of Snail and Twist, two upstream target proteins of $\mathrm{N}$-cadherin, vimentin and E-cadherin by western blotting. The results revealed that expression of both proteins was significantly decreased $(\mathrm{P}<0.05$; Fig. 4C and $\mathrm{D})$, suggesting that USP9X may be involved in regulating the migration and invasion of PANC-1 cells. As Snail and Twist were activated in pancreatic cancer cells, USP9X may be highly associated with disease progression by regulating the expression of downstream target proteins, including $\mathrm{N}$-cadherin, vimentin and E-cadherin.

Inhibition of USP9X induces apoptosis. The effect of USP9X on cell apoptosis was detected using flow cytometry. After the given plasmid was transfected into PANC-1 cells for $48 \mathrm{~h}$, shRNA-USP9X effectively inhibited USP9X expression compared with that noted in the control groups (Fig. 1). Then, the cells were stained with Annexin V-FITC/PI for assaying cell apoptosis. The apoptotic rate of PANC-1 cells increased after inhibition of USP9X expression in comparison with the uninduced counterparts (Fig. 5A and B), indicating that depletion of USP9X induced the apoptosis of PANC-1 cells. To confirm the effects of USP9X on the protein expression of survivin in PANC-1 cells, we measured survivin by western 


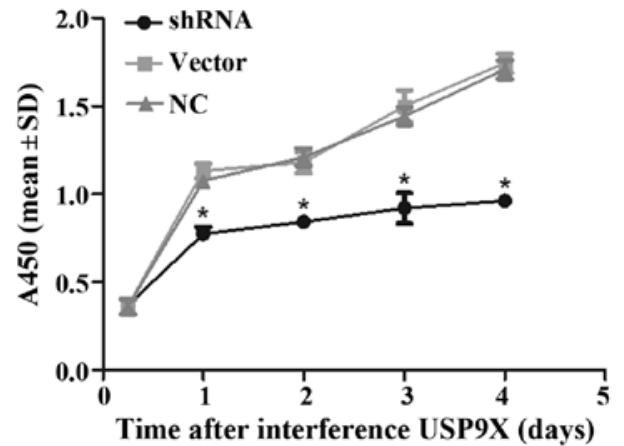

Figure 6. Downregulation of USP9X decreases cell proliferation. CCK-8 assays were performed at each time point to quantify cell viability of the PANC-1 cells transfected with USP9X shRNA, empty plasmid control (vector group) and normal control groups (NC group). The graph shows the changing trends among the three groups. All experiments were carried out in triplicate. Data are shown as mean $\pm \mathrm{SD} ;{ }^{*} \mathrm{P}<0.05$.

blotting. The results indicated that survivin expression in PANC-1 cells was markedly reduced compared with that noted in the control groups $(\mathrm{P}<0.05$, Fig. $5 \mathrm{C}$ and $\mathrm{D})$.

Depletion of USP9X impacts cell viability. As revealed by the CCK-8 assay, depletion of USP9X in PANC-1 cells resulted in decreased cell viability compared to the cell viability of another two groups of cells $(\mathrm{P}<0.05$; Fig. 6$)$.

\section{Discussion}

Pancreatic neoplasms are highly malignant tumors, and the prognosis of patients with PDAC is extremely poor, which is mostly ascribed to the high migration, invasiveness and high recurrence rate (22). Due to the broad range of its targets, the roles of USP9X are various and rely on different environments $(8,9,12,23,24)$. Abnormal expression of USP9X in several types of cancers has been reported $(8,11,23,24)$. Particularly, whether PDAC USP9X acts as a proto-oncogene or tumor-suppressor gene remains controversial (11), and the concrete effects of USP9X on migration, invasion and apoptosis of PDAC are still poorly understood.

Our experimental data support the role of USP9X as a growth promoter in tumor genesis and progression. As shown in Fig. 1A and B, the USP9X expression in human pancreatic cancer tissues was markedly increased compared with that noted in the adjacent non-tumor tissues. We believe that USP9X is positively correlated with the degree of tumor malignancy and may be closely related with pathophysiological progression of PDAC (Table I). Notably, downregulation of USP9X expression reduced the invasion and migration abilities of the PANC-1 cells (Fig. 3A-C). Depletion of USP9X reversed the EMT of PANC-1 cells, which is reflected in the changes of $\mathrm{N}$-cadherin, vimentin and E-cadherin expression. Moreover, cell apoptosis was increased after the downregulation of USP9X (Fig. 5A and B). The survivin expression was positively correlated with the USP9X level. Furthermore, inhibition of USP9X expression effectively decreased the viability of the PANC-1 cells (Fig. 6).

Notably, Cox et al demonstrated that USP9X acted principally as an oncogene in five tested PDAC cell lines (11).
However, Pérez-Mancera et al found that USP9X acted as a tumor-suppressor in a mouse model of pancreatic malignant tumors in which the USP9X expression was interfered at the early stage of tumor development by Sleeping Beauty transposon (12). In the present study, we believe that USP9X may be an oncogene in PANC-1 cells. Our results are similar to those of Cox et al. We suggest that downregulation of USP9X can inhibit the migration and invasion of pancreatic cancer PANC-1 cells and lead to a decrease in cell viability. The apoptosis rate of the PANC-1 cells was increased with the decrease in USP9X expression. The migratory ability of PANC-1 cells was weakened after downregulation of the expression of USP9X (Fig. 3A). We also found that our results differed from those of Perez-Mancera et al. The difference may be due to the pathological type of pancreatic cancer, the location of the pancreatic tumor or the difference in race. However, the specific reasons remain unclear and further research is needed.

Furthermore, we studied the specific proteins related to the migration and invasion functions of USP9X. We found that cell migration and invasion abilities were suppressed (Fig. 3A-C). Moreover, it is very possible that USP9X may affect the migration and invasion of PANC-1 cells by EMT. EMT is the process in which epithelial cells are transformed by specific procedures into a mesenchymal phenotype. Epithelial cells undergoing EMT lose polarity and some properties such as their connection to the basement membrane, which is an important process of tumor invasion and metastasis (3). It has also been confirmed that EMT is the molecular basis underlying the occurrence, invasion and metastasis of many tumors $(25,26)$. Furthermore, we examined the expression levels of $\mathrm{N}$-cadherin, vimentin and E-cadherin in PANC-1 cells by western blotting. However, the E-cadherin expression was significantly upregulated, while the expression of $\mathrm{N}$-cadherin and vimentin was downregulated in PANC-1 cells. This finding is consistent with other studies (27-29). We also tested Snail and Twist, which are the upstream target proteins of $\mathrm{N}$-cadherin, vimentin and E-cadherin. Twist belongs to the bHLH transcription factor family and plays an important role in tumor metastasis (15). Snail, a zinc finger transcription factor is combined with the promoter of the gene expression related to cell adhesion, and opens to regulate the transcription process (16). We found that the expression levels of both proteins were significantly downregulated (Fig. 4C and D). These results are consistent with previous research $(13,14,30)$. We conclude that USP9X is highly linked with disease formation and progression by activating EMT.

Moreover, we studied PANC-1 cell apoptosis after the expression of USP9X was silenced. The apoptotic rate of PANC-1 cells increased after the inhibition of USP9X expression compared with the uninduced groups (Fig. 5A and B). As reported, survivin is degraded by the ubiquitin proteasome pathway (20). Theoretically, downregulated expression of survivin can cause cell apoptosis. Thus, we detected the expression of survivin, which is the strongest apoptosis inhibitor (15). Survivin may inhibit apoptosis mainly by the following two ways: one way by directly inhibiting the activities of caspase-3 and -7, the end effectors of apoptosis protein, and another way by interacting with CDK-2 and CDK-4 and blocking the apoptotic signal transduction pathway (31). In PDAC, survivin is involved in many biological processes, such 
as gene transcription regulation, cell differentiation, tumor formation and metastasis $(32,33)$. We also found that the survivin expression in PANC-1 cells was markedly reduced after the inhibition of USP9X. The change in survivin expression was positively proportional to USP9X, but the concrete mechanism between them is still unclear and needs further study.

Taken together, USP9X participates in a variety of biological processes in pancreatic cancer cells. In the present study, we first demonstrated that USP9X activated the EMT pathway and altered cell migration and invasion abilities. We then explored the close relationship between USP9X and survivin in pancreatic cancer. Future studies are needed to reveal the specific mechanisms between Snail/Twist and USP9X, and between survivin and USP9X. Our findings may provide a novel strategy for the treatment of pancreatic cancer. Early inhibition of USP9X may delay the invasion and metastasis of PDAC, which makes it possible to improve the clinical outcomes of PDAC patients. However, our findings are not fully consistent with previous experts, thus deeper investigation is required to elucidate the significant role of USP9X in PDAC.

\section{References}

1. Siegel R, Ma J, Zou Z and Jemal A: Cancer statistics, 2014. CA Cancer J Clin 64: 9-29, 2014.

2. Hidalgo M: Pancreatic cancer. N Engl J Med 362: 1605-1617, 2010.

3. Bonnomet A, Brysse A, Tachsidis A, Waltham M, Thompson EW, Polette $\mathrm{M}$ and Gilles C: Epithelial-to-mesenchymal transitions and circulating tumor cells. J Mammary Gland Biol Neoplasia 15 261-273, 2010.

4. Kapuria V, Peterson LF, Fang D, Bornmann WG, Talpaz M and Donato NJ: Deubiquitinase inhibition by small-molecule WP1130 triggers aggresome formation and tumor cell apoptosis. Cancer Res 70: 9265-9276, 2010.

5. Kerscher O, Felberbaum R and Hochstrasser M: Modification of proteins by ubiquitin and ubiquitin-like proteins. Annu Rev Cell Dev Biol 22: 159-180, 2006.

6. Hussain S, Zhang Y and Galardy PJ: DUBs and cancer: The role of deubiquitinating enzymes as oncogenes, non-oncogenes and tumor suppressors. Cell Cycle 8: 1688-1697, 2009.

7. Reyes-Turcu FE, Ventii KH and Wilkinson KD: Regulation and cellular roles of ubiquitin-specific deubiquitinating enzymes. Annu Rev Biochem 78: 363-397, 2009.

8. Schwickart M, Huang X, Lill JR, Liu J, Ferrando R, French DM, Maecker H, O'Rourke K, Bazan F, Eastham-Anderson J, et al: Deubiquitinase USP9X stabilizes MCL1 and promotes tumour cell survival. Nature 463: 103-107, 2010.

9. Harris DR, Mims A and Bunz F: Genetic disruption of USP9X sensitizes colorectal cancer cells to 5-fluorouracil. Cancer Biol Ther 13: 1319-1324, 2012.

10. Liu H, Chen W, Liang C, Chen BW, Zhi X, Zhang S, Zheng X, Bai X and Liang T: WP1130 increases doxorubicin sensitivity in hepatocellular carcinoma cells through usp9x-dependent p53 degradation. Cancer Lett 361: 218-225, 2015.

11. Cox JL, Wilder PJ, Wuebben EL, Ouellette MM, Hollingsworth MA and Rizzino A: Context-dependent function of the deubiquitinating enzyme USP9X in pancreatic ductal adenocarcinoma. Cancer Biol Ther 15: 1042-1052, 2014.

12. Pérez-Mancera PA, Rust AG, van der Weyden L, Kristiansen G, Li A, Sarver AL, Silverstein KA, Grützmann R, Aust D, Rümmele $\mathrm{P}$, et al; Australian Pancreatic Cancer Genome Initiative: The deubiquitinase USP9X suppresses pancreatic ductal adenocarcinoma. Nature 486: 266-270, 2012.

13. De Craene B and Berx G: Regulatory networks defining EMT during cancer initiation and progression. Nat Rev Cancer 13 97-110, 2013
14. Yang $\mathbf{J}$ and Weinberg RA: Epithelial-mesenchymal transition: At the crossroads of development and tumor metastasis. Dev Cell 14: 818-829, 2008.

15. Khan MA, Chen HC, Zhang D and Fu J: Twist: A molecular target in cancer therapeutics. Tumour Biol 34: 2497-2506, 2013.

16. Smith BN and Odero-Marah VA: The role of Snail in prostate cancer. Cell Adhes Migr 6: 433-441, 2012.

17. Ambrosini G, Adida C and Altieri DC: A novel anti-apoptosis gene, survivin, expressed in cancer and lymphoma. Nat Med 3: 917-921, 1997.

18. Roca H, Varsos ZS, Mizutani K and Pienta KJ: CCL2, survivin and autophagy: New links with implications in human cancer. Autophagy 4: 969-971, 2008

19. Farnebo L, Tiefenböck K, Ansell A, Thunell LK, Garvin S and Roberg K: Strong expression of survivin is associated with positive response to radiotherapy and improved overall survival in head and neck squamous cell carcinoma patients. Int J Cancer 133: 1994-2003, 2013.

20. Liu YB, Gao X, Deeb D, Brigolin C, Zhang Y, Shaw J, Pindolia K and Gautam SC: Ubiquitin-proteasomal degradation of antiapoptotic survivin facilitates induction of apoptosis in prostate cancer cells by pristimerin. Int J Oncol 45: 1735-1741, 2014.

21. Peng J, Hu Q, Liu W, He X, Cui L, Chen X, Yang M, Liu H, Wei W, Liu S, et al: USP9X expression correlates with tumor progression and poor prognosis in esophageal squamous cell carcinoma. Diagn Pathol 8: 177-185, 2013.

22. Ahmad R: Cheema, Eileen M. O'Reilly. Management of metastatic pancreatic adenocarcinoma. Surg Clin North Am 7: 1391-1414, 2016.

23. Murtaza M, Jolly LA, Gecz J and Wood SA: La FAM fatale: USP9X in development and disease. Cell Mol Life Sci 72: 2075-2089, 2015.

24. Wang Y, Liu Y, Yang B, Cao H, Yang CX, Ouyang W, Zhang SM, Yang GF, Zhou FX, Zhou YF, et al: Elevated expression of USP9X correlates with poor prognosis in human non-small cell lung cancer. J Thorac Dis 7: 672-679, 2015.

25. Vermeulen L, Todaro M, de Sousa Mello F, Sprick MR, Kemper K, Perez Alea M, Richel DJ, Stassi G and Medema JP: Single-cell cloning of colon cancer stem cells reveals a multi-lineage differentiation capacity. Proc Natl Acad Sci USA 105: 13427-13432, 2008.

26. Findlay VJ, Wang C, Watson DK and Camp ER: Epithelial-tomesenchymal transition and the cancer stem cell phenotype: Insights from cancer biology with therapeutic implications for colorectal cancer. Cancer Gene Ther 21: 181-187, 2014.

27. von Burstin J, Eser S, Paul MC, Seidler B, Brandl M, Messer M, von Werder A, Schmidt A, Mages J, Pagel P, et al: E-cadherin regulates metastasis of pancreatic cancer in vivo and is suppressed by a SNAIL/HDAC1/HDAC2 repressor complex. Gastroentemlogy 137: 361-371, 2009

28. Lang SH, Hyde C, Reid IN, Hitchcock IS, Hart CA, Bryden AA, Villette JM, Stower MJ and Maitland NJ: Enhanced expression of vimentin in motile prostate cell lines and in poorly differentiated and metastatic prostate carcinoma. Prostate 52: 253-263, 2002.

29. LeeMY,ChouCY,TangMJandShenMR:Epithelial-mesenchymal transition in cervical cancer: Correlation with tumor progression, epidermal growth factor receptor overexpression, and snail up-regulation. Clin Cancer Res 14: 4743-4750, 2008.

30. Francí C, Takkunen M, Dave N, Alameda F, Gómez S, Rodríguez R, Escrivà M, Montserrat-Sentís B, Baró T, Garrido M, et al: Expression of Snail protein in tumor-stroma interface. Oncogene 25: 5134-5144, 2006.

31. Xu JH, Wang AX, Huang HZ, Wang JG, Pan CB and Zhang B: Survivin shRNA induces caspase-3-dependent apoptosis and enhances cisplatin sensitivity in squamous cell carcinoma of the tongue. Oncol Res 18: 377-385, 2010.

32. Lopes RB, Gangeswaran R, McNeish IA, Wang Y and Lemoine NR: Expression of the IAP protein family is dysregulated in pancreatic cancer cells and is important for resistance to chemotherapy. Int J Cancer 120: 2344-2352, 2007.

33. Jhala N, Jhala D, Vickers SM, Eltoum I, Batra SK, Manne U, Eloubeidi M, Jones JJ and Grizzle WE: Biomarkers in diagnosis of pancreatic carcinoma in fine-needle aspirates. Am J Clin Pathol 126: 572-579, 2006. 